\title{
MULTIPLE SOLUTIONS TO A NONLINEAR ELLIPTIC EQUATION INVOLVING PANEITZ TYPE OPERATORS
}

\author{
ABDALLAH EL HAMIDI
}

Received 12 May 2003

\begin{abstract}
This paper deals with an elliptic equation involving Paneitz type operators on compact Riemannian manifolds with concave-convex nonlinearities and a real parameter. Nonlocal and multiple existence results are established. Characteristic values of the real parameter are introduced and their role in the change of the energy sign and the existence of positive solutions are highlighted.
\end{abstract}

2000 Mathematics Subject Classification: 58J05, 35J35, 35J60.

1. Introduction. In this paper, we study a nonlinear elliptic equation involving Paneitz type operators on compact Riemannian manifolds. The nonlinearity considered here is concave-convex. The simultaneous effect of the concave and convex terms has been initially investigated by Ambrosetti et al. [1] in the Euclidian case for the Laplace operator. Since then, elliptic problems with this kind of nonlinearities were extensively studied by several authors with different classes of domains and with more general differential operators like the $p$-Laplacian. We can refer the reader to the valuable survey article by Ambrosetti et al. [2] and the references therein.

The aim of this paper is to establish nonlocal and multiple existence results (with respect to a real parameter) to an elliptic equation involving the Paneitz-Branson operator with concave-convex nonlinear terms. Also, characteristic values of the real parameter are introduced (under variational form) and some of their specific properties are carried out.

Now, let $(M, g)$ be a smooth 4-dimensional Riemannian manifold and let $S_{g}, R c_{g}$ be the scalar curvature and the Ricci curvature of $g$, respectively. The Paneitz operator, introduced by Paneitz [23], defined on $(M, g)$ is the fourth-order operator

$$
P_{g}^{4} u:=\Delta_{g}^{2} u-\operatorname{div}_{g}\left(\frac{2}{3} S_{g} g-2 R c_{g}\right) d u,
$$

where $\Delta_{g} u=-\operatorname{div}_{g} \nabla u$ is the Laplacian of $u$ and $d u$ is the differential of $u$, both with respect to the metric $g$.

The Paneitz operator was generalized to higher dimensions by Branson [5]. Given $(M, g)$, an $n$-dimensional Riemannian manifold $(n \geq 5)$, the Paneitz-Branson operator $P_{g}^{n}$ is defined as follows:

$$
P_{g}^{n} u:=\Delta_{g}^{2} u-\operatorname{div}_{g}\left(\frac{(n-2)^{2}+4}{2(n-1)(n-2)} S_{g} g-\frac{4}{n-2} R c_{g}\right) d u+\frac{n-4}{2} Q_{g}^{n} u
$$


where the $Q$-curvature $Q_{g}^{n}$ is given by

$$
Q_{g}^{n}=\frac{1}{2(n-1)} \Delta_{g} S_{g}+\frac{n^{3}-4 n^{2}+16 n-16}{8(n-1)^{2}(n-2)^{2}} S_{g}^{2}-\frac{2}{(n-2)^{2}}\left|R c_{g}\right|^{2} .
$$

Geometrically, the $Q$-curvature can be seen, for the Paneitz-Branson operator, as the analogue of the scalar curvature for the conformal Laplacian. Notice that when $(M, g)$ is Einstein, the Paneitz-Branson operator is reduced to

$$
P_{g}^{n} u=\Delta_{g}^{2} u+\alpha_{n} \Delta_{g} u+\beta_{n} u,
$$

where

$$
\alpha_{n}:=\frac{n^{2}-2 n-4}{2 n(n-1)} S_{g}, \quad \beta_{n}=\frac{(n-4)\left(n^{4}-4\right)}{16 n(n-1)^{2}} S_{g}^{2} .
$$

This is a special case of what one usually refers to as a Paneitz-Branson operator with constant coefficients, namely, an operator which is expressed as

$$
P_{g} u=\Delta_{g}^{2} u+\alpha \Delta_{g} u+\beta u,
$$

where $\alpha$ and $\beta$ are real numbers. In this direction, we can refer the reader to Djadli et al. [11], Esposito and Robert [14], Felli et al. [15], Hebey [17, 18], Hebey and Robert [19], and finally to Robert [25].

In this paper, we study the existence of multiple solutions to

$$
P_{g} u=\lambda a(x)|u|^{q-2} u+b(x)|u|^{r-2} u,
$$

with respect to the positive real parameter $\lambda$, where $(M, g)$ is a smooth compact Riemannian manifold of dimension $n \geq 5, a$ and $b$ are positive continuous functions on $M$. This problem is stated in the framework of the Sobolev space $H_{2}^{2}(M)$ consisting of functions $u$ in $L^{2}(M)$ which are such that $|\nabla u|$ and $\left|\nabla^{2} u\right|$ are also in $L^{2}(M)$. We limit ourselves to the case of subcritical concave-convex nonlinearity, that is,

$$
1<q<2<r<2^{\#}
$$

where $2^{\#}:=2 n /(n-4)$ is the critical exponent for the embedding of the Sobolev space $H_{2}^{2}$ in $L^{p}$-spaces. Also, we will assume along this work that [19]

$$
\frac{\alpha^{2}}{4} \geq \beta>0
$$

In this situation, it is clear that the Paneitz-Branson operator $P_{g}$ is coercive, that is, there is $C>0$ such that for any $u \in H_{2}^{2}(M)$,

$$
\int_{M}\left(P_{g} u\right) u d v_{g} \geq C\|u\|_{H_{2}^{2}(M)}^{2},
$$

where

$$
\|u\|_{H_{2}^{2}(M)}=\left\{\int_{M}\left(\left(\Delta_{g} u\right)^{2}+|\nabla u|^{2}+u^{2}\right) d v_{g}\right\}^{1 / 2}
$$


is the standard norm of $H_{2}^{2}(M)$. In the specific case where $(M, g)$ is Einstein, condition (1.9) is satisfied. Indeed,

$$
\frac{\alpha_{n}^{2}}{4}-\beta_{n}=\frac{S_{g}^{2}}{n^{2}(n-1)^{2}}
$$

For further detailed discussions on this subject, we refer the reader to Beckner [3], Branson et al. [6], Chang et al. [8], Chang and Yang [9, 10], and to Gursky [16] for the Paneitz operator. For the Paneitz-Branson operator, we mention the references described above $[11,14,15,17,18,19,25]$.

Hereafter, the space $H_{2}^{2}(M)$ will be endowed with the norm $\|\cdot\|$,

$$
\|u\|=\left(\int_{M}\left(P_{g} u\right) u d v_{g}\right)^{1 / 2}
$$

which is equivalent to norm $\|\cdot\|_{H_{2}^{2}(M)}$. Following standard notations, $\|\cdot\|_{p}$ will stand for the $L^{p}$-norm (with respect to the Riemannian measure $d v_{g}$ ).

Consider the following problem:

$$
\begin{gathered}
P_{g} u=\lambda a(x)|u|^{q-2} u+b(x)|u|^{r-2} u \quad \text { in } M, \\
1<q<2<r \leq 2^{\#} .
\end{gathered}
$$

For solutions of (1.14), we understand critical points of the associated Euler-Lagrange (energy) functional $E_{\lambda} \in C^{1}\left(H_{2}^{2}(M)\right)$, given by

$$
E_{\lambda}(u)=\frac{1}{2} P(u)-\frac{\lambda}{q} Q(u)-\frac{1}{r} R(u)
$$

where

$$
P(u)=\|u\|^{2}, \quad Q(u)=\int_{M} a|u|^{q} d v_{g}, \quad R(u)=\int_{M} b|u|^{r} d v_{g}
$$

In [4], Bernis et al. studied the following equation:

$$
\Delta^{2} u=\lambda|u|^{q-2} u+|u|^{2^{\#}-2} u
$$

in a smooth bounded domain in $\mathbb{R}^{n}$ with Dirichlet or Navier boundary conditions. Applying the Lusternik-Schnirelman theory, the authors showed the existence of infinitely many solutions for $\lambda$ small enough (a local result with respect to the parameter $\lambda)$. Using classical methods, the authors showed the existence of two positive solutions for $0<\lambda<\Lambda$, even in the supercritical case. Notice that our result concerning the existence of infinitely many solutions is not local. Indeed, we show the existence of two disjoint and infinite sets of solutions to (1.14) for $0<\lambda<\hat{\lambda}$, where $\hat{\lambda}>C\left(\|a\|_{\infty},\|b\|_{\infty}, q, r, M\right)>0$. The first set consists of solutions with negative energy, while the second set contains solutions with arbitrary energy. On the other hand, our 
proof concerning the existence of positive solutions gives a new argument for the construction of Palais-Smale sequences. Similar equation was studied also by the author [13] in the Euclidian case, with the $p$-Laplacian operator and nonlinear mixed boundary conditions.

We introduce the modified Euler-Lagrange functional $\widetilde{E}_{\lambda}[12,13,28]$, defined on $\mathbb{R} \times$ $H_{2}^{2}(M)$ by

$$
\widetilde{E}_{\lambda}(t, u):=E_{\lambda}(t u)
$$

If $u$ is an arbitrary element of $H_{2}^{2}(M), \partial_{t} \widetilde{E}_{\lambda}(\cdot, u)$ stands for the first derivative of the real-valued function: $t \mapsto \widetilde{E}_{\lambda}(t, u)$. Similarly, $\partial_{t t} \widetilde{E}_{\lambda}(\cdot, u)$ denotes the second derivative.

2. Existence of positive solutions. In this section, we show the existence of two "branches" of positive solutions to (1.14). The idea of the approach is as follows: for every $u \in H_{2}^{2}(M) \backslash\{0\}$ and $\lambda>0$, we determine the positive critical points (in terms of $u$ and $\lambda$ ) of the real-valued function $t \mapsto \widetilde{E}_{\lambda}(t, u)$. Then, the variable $t$ is substituted by these real critical points to obtain functionals, depending only on the variable $u$ (and the parameter $\lambda$ ), defined on the Nehari manifold [22]. One easily shows that these new functionals are bounded below, which implies that we can minimize to obtain possible critical points of the Euler-Lagrange $E_{\lambda}$ itself. However, the positivity of these critical points is not guaranteed. To show the existence of positive solutions, we carry out a new approach of an idea introduced, to our knowledge, by Djadli et al. [11] and Van der Vorst [27].

\subsection{Technical lemmas}

LEMMA 2.1. Let $u \in H_{2}^{2}(M) \backslash\{0\}$. There exists a unique $\lambda(u)>0$ such that the realvalued function $t \mapsto \partial_{t} \widetilde{E}_{\lambda}(t, u)$ has

(i) two positive zeros if $0<\lambda<\lambda(u)$,

(ii) one positive zero if $\lambda=\lambda(u)$,

(iii) no zeros if $\lambda>\lambda(u)$.

Proof. Let $u \in H_{2}^{2}(M) \backslash\{0\}$ and we write

$$
\partial_{t} \widetilde{E}_{\lambda}(t, u)=t^{q-1} \widetilde{F}_{\lambda}(t, u),
$$

where

$$
\widetilde{F}_{\lambda}(t, u)=t^{2-q} P(u)-\lambda Q(u)-t^{r-q} R(u) .
$$

It follows that

$$
\partial_{t t} \widetilde{E}_{\lambda}(t, u)=(q-1) t^{q-2} \widetilde{F}_{\lambda}(t, u)+t^{q-1} \partial_{t} \widetilde{F}_{\lambda}(t, u),
$$

with

$$
\partial_{t} \widetilde{F}_{\lambda}(t, u)=t^{2-q-1}\left\{(2-q) P(u)-(r-q) t^{r-2} R(u)\right\}
$$


The real-valued function $t \mapsto \widetilde{F}_{\lambda}(t, u)$ is increasing for $\left.t \in\right] 0, t(u)$ [, decreasing for $t \in$ ] $t(u),+\infty[$, and attains its unique maximum for $t=t(u)$, where

$$
t(u)=\left(\frac{2-q}{r-q} \frac{P(u)}{R(u)}\right)^{1 /(r-2)} .
$$

Therefore, the function $t \mapsto \widetilde{F}_{\lambda}(t, u)$ has

(i) two positive zeros if $\widetilde{F}_{\lambda}(t(u), u)>0$,

(ii) one positive zero if $\widetilde{F}_{\lambda}(t(u), u)=0$,

(iii) no zero if $\widetilde{F}_{\lambda}(t(u), u)<0$.

Moreover, a direct computation gives

$$
\tilde{F}_{\lambda}(t(u), u)=\frac{r-2}{2-q}\left(\frac{2-q}{r-q} \frac{P(u)}{R(u)}\right)^{(r-q) /(r-2)} R(u)-\lambda Q(u) .
$$

It follows that

$$
\begin{array}{ll}
\widetilde{F}_{\lambda}(t(u), u)>0 & \text { if } \lambda<\lambda(u), \\
\widetilde{F}_{\lambda}(t(u), u)=0 & \text { if } \lambda=\lambda(u), \\
\widetilde{F}_{\lambda}(t(u), u)<0 & \text { if } \lambda>\lambda(u),
\end{array}
$$

where

$$
\begin{gathered}
\lambda(u)=\hat{C} \frac{P^{(r-q) /(r-2)}(u)}{Q(u) R^{(2-q) /(r-2)}(u)}, \\
\hat{C}=\frac{r-2}{2-q}\left(\frac{2-q}{r-q}\right)^{(r-q) /(r-2)}
\end{gathered}
$$

Hence, if $\lambda \in] 0, \lambda(u)$ [, the real-valued function $t \mapsto \partial_{t} \widetilde{E}_{\lambda}(t, u)$ has two positive zeros which we will denote by $\underline{t}(u, \lambda)$ and $\bar{t}(u, \lambda)$. Notice that

$$
0<\underline{t}(u, \lambda)<t(u)<\bar{t}(u, \lambda) .
$$

Since, $\widetilde{F}_{\lambda}(\underline{t}(u, \lambda), u)=\widetilde{F}_{\lambda}(\bar{t}(u, \lambda), u)=0, \partial_{t} \widetilde{F}_{\lambda}(t, u)>0$ for $t<t(u)$, and $\partial_{t} \widetilde{F}_{\lambda}(t, u)<0$ for $t>t(u)$, we get

$$
\partial_{t t} \widetilde{E}_{\lambda}(\underline{t}(u, \lambda), u)>0, \quad \partial_{t t} \widetilde{E}_{\lambda}(\bar{t}(u, \lambda), u)<0 .
$$

Consequently, the real-valued function $t \mapsto \widetilde{E}_{\lambda}(t, u), t>0$, attains its unique local minimum at $t=\underline{t}(u, \lambda)$ and its unique local maximum at $t=\bar{t}(u, \lambda)$.

We specify that for every $u \in H_{2}^{2}(M) \backslash\{0\}$ and $\left.\lambda \in\right] 0, \lambda(u)[, \underline{t}(u, \lambda) u$ and $\bar{t}(u, \lambda) u$ belong to the Nehari manifold [22] defined by

$$
\mathcal{N}:=\left\{v \in H_{2}^{2}(M) \backslash\{0\}: E_{\lambda}^{\prime}(v) v=0\right\}
$$


At this stage, we introduce the characteristic value

$$
\hat{\lambda}:=\inf _{u \in H_{2}^{2}(M) \backslash\{0\}} \lambda(u)
$$

of the parameter $\lambda$. We will show below that problem (1.14) possesses two "branches" of solutions for $\lambda \in] 0, \hat{\lambda}$ [. It is interesting to remark that this result is not local with respect to the parameter $\lambda$. Indeed, let $S_{q}(M)$ and $S_{r}(M)$ be the best Sobolev constants of the embeddings $H_{2}^{2}(M) \subset L^{q}(M)$ and $H_{2}^{2}(M) \subset L^{r}(M)$, respectively. Then,

$$
\begin{aligned}
\hat{\lambda} & =\hat{C} \inf _{u \in H_{2}^{2}(M) \backslash\{0\}}\left(\frac{[P(u)]^{q / 2}}{Q(u)}\right)\left(\frac{[P(u)]^{r / 2}}{R(u)}\right) \\
& \geq \frac{\hat{C}}{\max _{x \in M} a(x)\left[\max _{x \in M} b(x)\right]^{(2-q) /(r-2)}}\left[S_{q}(M)\right]^{q / 2}\left[S_{r}(M)\right]^{r(2-q) / 2(r-2)} \\
& >0
\end{aligned}
$$

Finally, remark that the function $\lambda(\cdot)$ is homogeneous on $H_{2}^{2}(M) \backslash\{0\}$.

Now, we show an interesting lemma which will be very useful below to construct positive solutions from solutions with arbitrary sign.

LEMMA 2.2. Let $\lambda \in] 0, \hat{\lambda}\left[, v\right.$ and $w \in H_{2}^{2}(M) \backslash\{0\}$. If

$$
\begin{gathered}
\|v\|=\|w\|, \\
|v| \leq|w| \quad \text { in } M,
\end{gathered}
$$

then

$$
\tilde{E}_{\lambda}(\underline{t}(v, \lambda), v) \geq \tilde{E}_{\lambda}(\underline{t}(w, \lambda), w), \quad \tilde{E}_{\lambda}(\bar{t}(v, \lambda), v) \geq \widetilde{E}_{\lambda}(\bar{t}(w, \lambda), w) .
$$

Proof. Without loss of generality, we can assume $\|v\|=\|w\|=1$. Consider the application

$$
\begin{gathered}
\left.\Phi_{\lambda}:\right] 0,+\infty\left[^{3} \longrightarrow \mathbb{R}\right. \\
(\xi, \theta, \sigma) \longmapsto \frac{\xi^{2}}{2}-\frac{\lambda \theta \xi^{q}}{q}-\frac{\sigma \xi^{r}}{r} .
\end{gathered}
$$

According to the study done in Lemma 2.1, for every $\theta>0, \sigma>0$, and $\lambda$ satisfying

$$
0<\lambda<\frac{\hat{C}}{\theta \sigma^{(2-q) /(r-2)}},
$$

the real-valued function $\Phi_{\lambda}(\cdot, \theta, \sigma)$ starts from $\Phi_{\lambda}(0, \theta, \sigma)=0$, decreases to reach its unique local minimum for $\xi=\underline{\xi}(\theta, \sigma)$, increases to reach its unique local maximum for $\xi=\bar{\xi}(\theta, \sigma)$, and finally decreases towards $-\infty$ when $\xi$ goes to $+\infty$. Using the fact that

$$
\frac{\partial^{2} \Phi_{\lambda}}{\partial \xi^{2}}(\underline{\xi}(\theta, \sigma), \theta, \sigma)>0, \quad \frac{\partial^{2} \Phi_{\lambda}}{\partial \xi^{2}}(\bar{\xi}(\theta, \sigma), \theta, \sigma)<0,
$$


the implicit function theorem implies that $\underline{\xi}(\cdot, \cdot)$ and $\bar{\xi}(\cdot, \cdot)$ are smooth in $\theta$ and $\sigma$. On the other hand, direct computations show that

$$
\begin{gathered}
\frac{\partial}{\partial \theta}\left[\Phi_{\lambda}(\underline{\xi}(\theta, \sigma), \theta, \sigma)\right]=-\frac{\lambda}{q}(\underline{\xi}(\theta, \sigma))^{q}<0, \\
\frac{\partial}{\partial \sigma}\left[\Phi_{\lambda}(\underline{\xi}(\theta, \sigma), \theta, \sigma)\right]=-\frac{1}{r}(\underline{\xi}(\theta, \sigma))^{r}<0 .
\end{gathered}
$$

Similarly, we have

$$
\begin{aligned}
& \frac{\partial}{\partial \theta}\left[\Phi_{\lambda}(\bar{\xi}(\theta, \sigma), \theta, \sigma)\right]=-\frac{\lambda}{q}(\bar{\xi}(\theta, \sigma))^{q}<0, \\
& \frac{\partial}{\partial \sigma}\left[\Phi_{\lambda}(\bar{\xi}(\theta, \sigma), \theta, \sigma)\right]=-\frac{1}{r}(\bar{\xi}(\theta, \sigma))^{r}<0 .
\end{aligned}
$$

This means that the critical values $\Phi_{\lambda}(\xi(\theta, \sigma), \theta, \sigma)$ and $\Phi_{\lambda}(\bar{\xi}(\theta, \sigma), \theta, \sigma)$ decrease when $\theta$ or $\sigma$ increases.

Now, let $0<\theta_{1} \leq \theta_{2}, 0<\sigma_{1} \leq \sigma_{2}$, and $\lambda>0$ such that

$$
0<\lambda<\frac{\hat{C}}{\theta_{i} \sigma_{i}^{(2-q) /(r-2)}}, \quad i=1,2 .
$$

The relations (2.22) can be rewritten as $\left(\theta_{i}, \sigma_{i}\right) \in \Omega_{\lambda}, i=1,2$, where $\Omega_{\lambda}$ denotes the subset of $\mathbb{R}^{2}$ defined by

$$
\Omega_{\lambda}=\{(\theta, \sigma) \in] 0,+\infty\left[^{2} ; \theta \sigma^{(2-q) /(r-2)}<\frac{\hat{C}}{\lambda}\right\}
$$

Consider the curve (path) $\gamma \subset \Omega_{\lambda}$ defined by

$$
\gamma:=\left\{\left(\theta, \sigma_{1}\right) ; \theta_{1} \leq \theta \leq \theta_{2}\right\} \cup\left\{\left(\theta_{2}, \sigma\right) ; \sigma_{1} \leq \sigma \leq \sigma_{2}\right\}
$$

connecting $\left(\theta_{1}, \sigma_{1}\right)$ to $\left(\theta_{2}, \sigma_{2}\right)$. It follows from above that the functions $(\theta, \sigma) \mapsto \Phi_{\lambda}(\xi(\theta$, $\sigma), \theta, \sigma)$ and $(\theta, \sigma) \mapsto \Phi_{\lambda}(\bar{\xi}(\theta, \sigma), \theta, \sigma)$ decrease when $(\theta, \sigma)$ describes the path $\gamma$ from $\left(\theta_{1}, \sigma_{1}\right)$ to $\left(\theta_{2}, \sigma_{2}\right)$. Therefore, we get

$$
\begin{aligned}
& \Phi_{\lambda}\left(\underline{\xi}\left(\theta_{1}, \sigma_{1}\right), \theta_{1}, \sigma_{1}\right) \geq \Phi_{\lambda}\left(\underline{\xi}\left(\theta_{2}, \sigma_{2}\right), \theta_{2}, \sigma_{2}\right), \\
& \Phi_{\lambda}\left(\bar{\xi}\left(\theta_{1}, \sigma_{1}\right), \theta_{1}, \sigma_{1}\right) \geq \Phi_{\lambda}\left(\bar{\xi}\left(\theta_{2}, \sigma_{2}\right), \theta_{2}, \sigma_{2}\right) .
\end{aligned}
$$

Here, $Q(v), R(v), Q(w)$, and $R(w)$ will play the role of $\theta_{1}, \sigma_{1}, \theta_{2}$, and $\sigma_{2}$, respectively. Moreover, specify that for every $u \in H_{2}^{2}(M)$ such that $\|u\|=1$, we have $\widetilde{E}_{\lambda}(t, u)=$ $\Phi_{\lambda}(t, Q(u), R(u))$. Hence, we conclude that

$$
\widetilde{E}_{\lambda}(\underline{t}(v, \lambda), v) \geq \widetilde{E}_{\lambda}(\underline{t}(w, \lambda), w), \quad \widetilde{E}_{\lambda}(\bar{t}(v, \lambda), v) \geq \widetilde{E}_{\lambda}(\bar{t}(w, \lambda), w) .
$$

This achieves the proof. 
We now show that the infimum of $\widetilde{E}_{\lambda}(\underline{t}(u, \lambda), u)$ and $\widetilde{E}_{\lambda}(\bar{t}(u, \lambda), u)$, when $u$ describes $H_{2}^{2}(M) \backslash\{0\}$, exists in $\mathbb{R}$. Indeed, since $\partial_{t} \widetilde{E}_{\lambda}(\underline{t}(u, \lambda), u)=0$, it follows that

$$
P(\underline{t}(u, \lambda) u)=\lambda Q(\underline{t}(u, \lambda) u)+R(\underline{t}(u, \lambda) u) .
$$

Then

$$
E_{\lambda}(\underline{t}(u, \lambda) u)=\left(\frac{1}{2}-\frac{1}{r}\right) R(\underline{t}(u, \lambda) u)-\lambda\left(\frac{1}{q}-\frac{1}{2}\right) Q(\underline{t}(u, \lambda) u)
$$

Using the fact that $1<q<2<r$, we get

$$
\lim _{\|\underline{t}(u, \lambda) u\| \rightarrow \infty} E_{\lambda}(\underline{t}(u, \lambda) u)=+\infty
$$

which implies that the function $u \mapsto E_{\lambda}(\underline{t}(u, \lambda) u)$, defined on $H_{2}^{2}(M) \backslash\{0\}$, is bounded below. In the same way, we get that the function $u \mapsto E_{\lambda}(\bar{t}(u, \lambda) u)$, defined on $H_{2}^{2}(M) \backslash$ $\{0\}$, is bounded below. Therefore, if we define

$$
\begin{aligned}
& \underline{\alpha}(\lambda)=\inf _{u \in H_{2}^{2}(M) \backslash\{0\}} \tilde{E}_{\lambda}(\underline{t}(u, \lambda), u), \\
& \bar{\alpha}(\lambda)=\inf _{u \in H_{2}^{2}(M) \backslash\{0\}} \widetilde{E}_{\lambda}(\bar{t}(u, \lambda), u),
\end{aligned}
$$

we have the following lemma.

LEMMA 2.3. Let $\left(u_{n}\right) \subset H_{2}^{2}(M) \backslash\{0\}$ be a minimizing sequence of (2.30) and $\underline{v}_{n}:=$ $\underline{t}\left(u_{n}, \lambda\right) u_{n}$. Then

(a) $\lim \sup _{n \rightarrow+\infty}\left\|\underline{v}_{n}\right\|<+\infty$,

(b) $\liminf { }_{n \rightarrow+\infty}\left\|\underline{v}_{n}\right\|>0$.

Similarly, let $\left(u_{n}\right) \subset H_{2}^{2}(M) \backslash\{0\}$ be a minimizing sequence of (2.31) and $\bar{v}_{n}:=$ $\bar{t}\left(u_{n}, \lambda\right) u_{n}$. Then

(c) $\limsup _{n \rightarrow+\infty}\left\|\bar{v}_{n}\right\|<+\infty$,

(d) $\liminf _{n \rightarrow+\infty}\left\|\bar{v}_{n}\right\|>0$.

Proof. (a) Let $\left(u_{n}\right) \subset H_{2}^{2}(M) \backslash\{0\}$ be a minimizing sequence of (2.30). Since $\partial_{t} \widetilde{E}_{\lambda}\left(\underline{t}\left(u_{n}, \lambda\right), u_{n}\right)=0$, it follows that

$$
P\left(\underline{v}_{n}\right)=\lambda Q\left(\underline{v}_{n}\right)+R\left(\underline{v}_{n}\right)
$$

Similarly, since $\partial_{t t} \widetilde{E}_{\lambda}\left(\underline{t}\left(u_{n}, \lambda\right), u_{n}\right)>0$, it follows that

$$
P\left(\underline{v}_{n}\right)-\lambda(q-1) Q\left(\underline{v}_{n}\right)-(r-1) R\left(\underline{v}_{n}\right)>0 .
$$

Combining (2.32) and (2.33), we obtain

$$
E_{\lambda}\left(\underline{v}_{n}\right):=\frac{1}{2} P\left(\underline{v}_{n}\right)-\frac{\lambda}{q} Q\left(\underline{v}_{n}\right)-\frac{1}{r} R\left(\underline{v}_{n}\right)<0,
$$


for every $n$. Suppose that there is a subsequence of $\left(\underline{v}_{n}\right)$, still denoted by $\left(\underline{v}_{n}\right)$, such that $\lim _{n \rightarrow+\infty}\left\|\underline{v}_{n}\right\|=+\infty$. Since $M$ is compact and $a, b$ are positive and continuous on $M$, then there is $C>0$ such that $Q\left(\underline{v}_{n}\right) \leq C R\left(\underline{v}_{n}\right)$ for every $n$. Therefore, $\lim _{n \rightarrow+\infty} R\left(\underline{v}_{n}\right)=$ $+\infty$. Using the fact that $0<q<r$, we get $Q\left(\underline{v}_{n}\right)=\mathrm{o}_{n}\left(R\left(\underline{v}_{n}\right)\right)$, and consequently

$$
P\left(\underline{v}_{n}\right)=R\left(\underline{v}_{n}\right)\left(1+\mathrm{o}_{n}(1)\right)
$$

Thus,

$$
E_{\lambda}\left(\underline{v}_{n}\right)=R\left(\underline{v}_{n}\right)\left(\frac{1}{2}-\frac{1}{r}+\mathrm{o}_{n}(1)\right)
$$

which implies that $E_{\lambda}\left(\underline{v}_{n}\right)$ tends to $+\infty$ as $n$ goes to $+\infty$, and this is impossible. We conclude that $\lim \sup _{n \rightarrow+\infty}\left\|\underline{v}_{n}\right\|<+\infty$.

(c) The same arguments with a minimizing sequence $\left(u_{n}\right)$ of (2.31) show that $\limsup \sup _{n \rightarrow+\infty}\left\|\bar{v}_{n}\right\|<+\infty$.

(b) Let $\left(u_{n}\right) \subset H_{2}^{2}(M) \backslash\{0\}$ be a minimizing sequence of (2.30) and suppose that there is a subsequence of $\left(\underline{v}_{n}\right)$, still denoted by $\left(\underline{v}_{n}\right)$, such that $\lim _{n \rightarrow+\infty}\left\|\underline{v}_{n}\right\|=0$. It follows that $\lim _{n \rightarrow+\infty} E_{\lambda}\left(\underline{v}_{n}\right)=0$, that is, $\underline{\alpha}(\lambda)=0$, which is impossible since $\widetilde{E}_{\lambda}\left(\underline{t}\left(u_{n}, \lambda\right), u_{n}\right)<$ 0 , for every $n$.

(d) Let $\left(u_{n}\right) \subset H_{2}^{2}(M) \backslash\{0\}$ be a mini mizing sequence of (2.31). Since $\partial_{t} \widetilde{E}_{\lambda}\left(\bar{t}\left(u_{n}, \lambda\right)\right.$, $\left.u_{n}\right)=0$ and $\partial_{t t} \widetilde{E}_{\lambda}\left(\bar{t}\left(u_{n}, \lambda\right), u_{n}\right)<0$, it follows that

$$
\begin{gathered}
P\left(\bar{v}_{n}\right)-\lambda Q\left(\bar{v}_{n}\right)-R\left(\bar{v}_{n}\right)=0, \\
P\left(\bar{v}_{n}\right)-\lambda(q-1) Q\left(\bar{v}_{n}\right)-(r-1) R\left(\bar{v}_{n}\right)<0 .
\end{gathered}
$$

Combining the last two inequalities, we obtain, for every $n$,

$$
(2-q) P\left(\bar{v}_{n}\right)<(r-q) R\left(\bar{v}_{n}\right) \leq C^{\prime} P\left(\bar{v}_{n}\right)
$$

via the continuous embedding $H_{2}^{2}(M) \subset L^{r}(M)$. Then $(2-q) \leq C^{\prime}\left\|\bar{v}_{n}\right\|^{r-2}$. Now, suppose that there is a subsequence of $\left(\bar{v}_{n}\right)$, still denoted by $\left(\bar{v}_{n}\right)$, such that $\lim _{n \rightarrow+\infty}\left\|\bar{v}_{n}\right\|=$ 0 . This implies that $2-q \leq 0$, which is impossible.

At this stage, we give an invariance result satisfied by $\underline{t}(u, \lambda)$ and $\bar{t}(u, \lambda)$ with respect to $u$. Indeed, for every real number $\gamma>0$, we have

$$
\begin{gathered}
\widetilde{E}_{\lambda}\left(\gamma t, \frac{u}{\gamma}\right)=\widetilde{E}_{\lambda}(t, u), \\
\partial_{t} \widetilde{E}_{\lambda}\left(\gamma t, \frac{u}{\gamma}\right)=\frac{1}{\gamma} \partial_{t} \widetilde{E}_{\lambda}(t, u), \\
\partial_{t t} \widetilde{E}_{\lambda}\left(\gamma t, \frac{u}{\gamma}\right)=\frac{1}{\gamma^{2}} \partial_{t t} \widetilde{E}_{\lambda}(t, u) .
\end{gathered}
$$


The uniqueness of the local minimum and the local maximum of the real-valued function $t \mapsto \widetilde{E}_{\lambda}(t, u), t>0, u \in H_{2}^{2}(M) \backslash\{0\}$, implies that

$$
\begin{aligned}
& \underline{t}(u, \lambda)=\frac{1}{\gamma} \underline{t}\left(\frac{u}{\gamma}, \lambda\right), \\
& \bar{t}(u, \lambda)=\frac{1}{\gamma} \bar{t}\left(\frac{u}{\gamma}, \lambda\right) .
\end{aligned}
$$

Hence, we get

$$
\begin{aligned}
& \underline{\alpha}(\lambda)=\inf _{u \in \mathbb{S}} \widetilde{E}_{\lambda}(\underline{t}(u, \lambda), u), \\
& \bar{\alpha}(\lambda)=\inf _{u \in \mathbb{S}} \widetilde{E}_{\lambda}(\bar{t}(u, \lambda), u),
\end{aligned}
$$

where $\mathbb{S}$ is the unit sphere of $H_{2}^{2}(M)$.

\subsection{Palais-Smale sequences and positive solutions}

THEOREM 2.4. Let $\left(u_{n}\right) \subset \mathbb{S}$ be a minimizing sequence of (2.41) (resp., of (2.42)). Then, $\left(\underline{v}_{n}\right):=\left(\underline{t}\left(u_{n}, \lambda\right) u_{n}\right)$ (resp., $\left.\left(\bar{v}_{n}\right):=\left(\bar{t}\left(u_{n}, \lambda\right) u_{n}\right)\right)$ is a Palais-Smale sequence for the functional $E_{\lambda}$.

Proof. Let $\left(u_{n}\right) \subset \mathbb{S}$ be a minimizing sequence of (2.41). According to the previous lemma, $\left(\underline{v}_{n}\right)$ is bounded in $H_{2}^{2}(M)$. Moreover, for every $u \in H_{2}^{2}(M) \backslash\{0\}$ and $\left.\lambda \in\right] 0, \hat{\lambda}$ [, we know that $\partial_{t} \widetilde{E}_{\lambda}(\underline{t}(u, \lambda), u)=0$ and $\partial_{t t} \widetilde{E}_{\lambda}(\underline{t}(u, \lambda), u) \neq 0$. The implicit function theorem implies that $\underline{t}(u, \lambda)$ is $C^{1}$ with respect to $u$ since $\widetilde{E}$ is. We introduce the $C^{1}$ functional $\underline{\mathscr{E}} \lambda$ defined on $\mathbb{S}$ by

$$
\underline{\mathscr{E}}_{\lambda}(u)=\widetilde{E}_{\lambda}(\underline{t}(u, \lambda), u)=E_{\lambda}(\underline{t}(u, \lambda) u) .
$$

Then

$$
\underline{\alpha}(\lambda)=\inf _{u \in \mathbb{S}} \mathscr{\mathscr { E }}_{\lambda}(u), \quad \lim _{n \rightarrow+\infty} \underline{\mathscr{E}}_{\lambda}\left(u_{n}\right)=\underline{\alpha}(\lambda) .
$$

Using the Ekeland variational principle on the complete manifold $(\mathbb{S},\|\cdot\|)$ to the functional $\underline{\mathscr{E}}_{\lambda}$, we conclude that

$$
\left|\underline{\mathscr{\ell}}_{\lambda}^{\prime}\left(u_{n}\right)\left(\varphi_{n}\right)\right| \leq \frac{1}{n}\left\|\varphi_{n}\right\|, \quad \text { for every } \varphi_{n} \in \mathrm{T}_{u_{n}} \mathbb{S}
$$

where $\mathrm{T}_{u_{n}} \mathbb{S}$ is the tangent space to $\mathbb{S}$ at the point $u_{n}$. Moreover, for every $\varphi_{n} \in \mathrm{T}_{u_{n}} \mathbb{S}$, one has

$$
\begin{aligned}
\underline{\mathscr{E}}_{\lambda}^{\prime}\left(u_{n}\right)\left(\varphi_{n}\right) & =\partial_{t} \widetilde{E}_{\lambda}\left(\underline{t}\left(u_{n}, \lambda\right), u_{n}\right) \underline{t}^{\prime}\left(u_{n}, \lambda\right)\left(\varphi_{n}\right)+\partial_{u} \widetilde{E}_{\lambda}\left(\underline{t}\left(u_{n}, \lambda\right), u_{n}\right)\left(\varphi_{n}\right) \\
& =\partial_{u} \widetilde{E}_{\lambda}\left(\underline{t}\left(u_{n}, \lambda\right), u_{n}\right)\left(\varphi_{n}\right),
\end{aligned}
$$


since $\partial_{t} \widetilde{E}_{\lambda}\left(\underline{t}\left(u_{n}, \lambda\right), u_{n}\right)=0$, where $\underline{t}^{\prime}\left(u_{n}, \lambda\right)$ denotes the derivative of $\underline{t}(\cdot, \lambda)$ with respect to its first variable at the point $\left(u_{n}, \lambda\right)$.

On the other hand, let

$$
\begin{gathered}
\pi: H_{2}^{2}(M) \backslash\{0\} \longrightarrow \mathbb{R} \times \mathbb{S}, \\
u \longmapsto\left(\|u\|, \frac{u}{\|u\|}\right):=\left(\pi_{1}(u), \pi_{2}(u)\right) .
\end{gathered}
$$

Applying Hölder's inequality, we get for every $(u, \varphi) \in\left(H_{2}^{2}(M) \backslash\{0\}\right) \times H_{2}^{2}(M)$,

$$
\begin{gathered}
\left|\pi_{1}^{\prime}(u)(\varphi)\right| \leq\|\varphi\|, \\
\left\|\pi_{2}^{\prime}(u)(\varphi)\right\| \leq 2 \frac{\|\varphi\|}{\|u\|} .
\end{gathered}
$$

From Lemma 2.3, there is a positive constant $C$ such that

$$
\underline{t}\left(u_{n}, \lambda\right) \geq C, \quad \forall n \in \mathbb{N} .
$$

Then, for every $\varphi \in H_{2}^{2}(M)$, there are $\varphi_{n}^{1}=\pi_{1}^{\prime}\left(u_{n}\right)(\varphi) \in \mathbb{R}$ and $\varphi_{n}^{2}=\pi_{2}^{\prime}\left(u_{n}\right)(\varphi) \in$ $\mathrm{T}_{u_{n}} \mathbb{S}$ such that $\left|\varphi_{n}^{1}\right| \leq\|\varphi\|,\left\|\varphi_{n}^{2}\right\| \leq(2 / C)\|\varphi\|$. This allows to obtain

$$
\begin{aligned}
E_{\lambda}^{\prime}\left(\underline{t}\left(u_{n}, \lambda\right) u_{n}\right)(\varphi) & =\partial_{t} \widetilde{E}_{\lambda}\left(\underline{t}\left(u_{n}, \lambda\right), u_{n}\right)\left(\varphi_{n}^{1}\right)+\partial_{u} \widetilde{E}_{\lambda}\left(\underline{t}\left(u_{n}, \lambda\right), u_{n}\right)\left(\varphi_{n}^{2}\right) \\
& =\partial_{u} \widetilde{E}_{\lambda}\left(\underline{t}\left(u_{n}, \lambda\right), u_{n}\right)\left(\varphi_{n}^{2}\right) \\
& =\underline{\mathscr{E}}_{\lambda}^{\prime}\left(u_{n}\right)\left(\varphi_{n}^{2}\right) .
\end{aligned}
$$

More precisely, we get

$$
E_{\lambda}^{\prime}\left(\underline{t}\left(u_{n}, \lambda\right) u_{n}\right)(\varphi) \leq \frac{1}{n}\left\|\varphi_{n}^{2}\right\| \leq \frac{2}{n C}\|\varphi\| .
$$

In other words,

$$
\lim _{n \rightarrow \infty}\left\|E_{\lambda}^{\prime}\left(\underline{v}_{n}\right)\right\|_{*}=0
$$

which achieves the first claim. The same arguments can be used to show that $\left(\bar{v}_{n}\right)$ is a Palais-Smale sequence for the functional $E_{\lambda}$. This ends the proof.

THEOREM 2.5. Let $1<q<2<r<2^{\#}$ and $\left.\lambda \in\right] 0, \hat{\lambda}[$. Then problem (1.14) has at least two positive solutions.

Proof. We will adopt the notations used in the previous lemmas. As mentioned in Theorem 2.4, $E_{\lambda}\left(\underline{v}_{n}\right)$ converges to $\underline{\alpha}(\lambda),\left\|E_{\lambda}^{\prime}\left(\underline{v}_{n}\right)\right\|_{*}$ converges to 0 as $n$ tends to $+\infty$, and $\left(\underline{v}_{n}\right)$ is bounded in $H_{2}^{2}(M)$. Passing, if necessary, to a subsequence, we have

$$
\begin{gathered}
\underline{v}_{n}-\underline{v} \quad \text { in } H_{2}^{2}(M), \\
\underline{v}_{n} \longrightarrow \underline{v} \quad \text { in } L^{r}(M)\left(\operatorname{also} \text { in } L^{q}(M)\right), \\
\underline{v}_{n} \longrightarrow \underline{v} \quad \text { a.e. in } M .
\end{gathered}
$$


Let $w_{n}=\underline{v}_{n}-\underline{v}$; then, using a lemma due to Brézis and Lieb [7], we get

$$
\begin{aligned}
& P\left(w_{n}\right)=P\left(\underline{v}_{n}\right)-P(\underline{v})+\mathrm{o}_{n}(1), \\
& Q\left(w_{n}\right)=Q\left(\underline{v}_{n}\right)-Q(\underline{v})+\mathrm{o}_{n}(1), \\
& R\left(w_{n}\right)=R\left(\underline{v}_{n}\right)-R(\underline{v})+\mathrm{o}_{n}(1) .
\end{aligned}
$$

It follows that

$$
\begin{aligned}
& E_{\lambda}\left(w_{n}\right)=E_{\lambda}\left(\underline{v}_{n}\right)-E_{\lambda}(\underline{v})+\mathrm{o}_{n}(1), \\
& E_{\lambda}^{\prime}\left(w_{n}\right)=E_{\lambda}^{\prime}\left(\underline{v}_{n}\right)-E_{\lambda}^{\prime}(\underline{v})+\mathrm{o}_{n}(1),
\end{aligned}
$$

and consequently $E_{\lambda}^{\prime}\left(w_{n}\right) w_{n} \rightarrow 0$ as $n \rightarrow+\infty$, which implies that

$$
\left\|w_{n}\right\|^{2}=\lambda Q\left(w_{n}\right)+R\left(w_{n}\right)+\mathrm{o}_{n}(1)
$$

Therefore, $\left\|w_{n}\right\| \rightarrow 0$ as $n \rightarrow+\infty$. Hence, $\underline{v}_{n}$ converges strongly to some $\underline{v}$ in $H_{2}^{2}(M) \backslash$ $\{0\}$. In other words, there is $\underline{v} \in H_{2}^{2}(M) \backslash\{0\}$ such that

$$
\bar{t}\left(u_{n}, \lambda\right) u_{n} \longrightarrow \underline{v} \quad \text { in } H_{2}^{2}(M)
$$

Since $\left(u_{n}\right)$ belongs to the unit sphere $\mathbb{S}$, then

$$
\begin{gathered}
\bar{t}\left(u_{n}, \lambda\right) \longrightarrow \underline{t}:=\|\underline{v}\|>0, \\
u_{n} \longrightarrow \underline{u}:=\underline{v} /\|\underline{v}\| \quad \text { in } H_{2}^{2}(M) .
\end{gathered}
$$

Notice that

$$
\begin{gathered}
\partial_{t} \widetilde{E}_{\lambda}(\underline{t}, \underline{u})=\lim _{n \rightarrow \infty} \partial_{t} \widetilde{E}_{\lambda}\left(\underline{t}\left(u_{n}, \lambda\right), u_{n}\right)=0, \\
\partial_{t t} \widetilde{E}_{\lambda}(\underline{t}, \underline{u})=\lim _{n \rightarrow \infty} \partial_{t t} \widetilde{E}_{\lambda}\left(\underline{t}\left(u_{n}, \lambda\right), u_{n}\right) \geq 0 .
\end{gathered}
$$

But $\partial_{t t} \widetilde{E}_{\lambda}(\underline{t}, \underline{u})=0$ cannot occur since $0<\lambda<\hat{\lambda}$ and because of Lemma 2.1. Hence,

$$
\begin{gathered}
\partial_{t} \widetilde{E}_{\lambda}(\underline{t}, \underline{u})=\lim _{n \rightarrow \infty} \partial_{t} \widetilde{E}_{\lambda}\left(\underline{t}\left(u_{n}, \lambda\right), u_{n}\right)=0, \\
\partial_{t t} \widetilde{E}_{\lambda}(\underline{t}, \underline{u})=\lim _{n \rightarrow \infty} \partial_{t t} \widetilde{E}_{\lambda}\left(\underline{t}\left(u_{n}, \lambda\right), u_{n}\right)>0,
\end{gathered}
$$

which implies that

$$
\underline{t}=\underline{t}(\underline{u}, \lambda) .
$$

Notice that $\underline{u}$ is not necessarily positive. At this stage, let $\underline{w} \in H_{2}^{2}(M)$ be the solution of

$$
\left(\Delta_{g}+\frac{\alpha}{2}\right) \underline{w}=\left|\left(\Delta_{g}+\frac{\alpha}{2}\right) \underline{u}\right| \quad \text { in } M
$$


It is clear that $\left(\Delta_{g}+\alpha / 2\right)(\underline{w}-\underline{u}) \geq 0$ and $\left(\Delta_{g}+\alpha / 2\right)(\underline{w}+\underline{u}) \geq 0$. Since the manifold $M$ is compact, the maximum principle for the operator $\Delta_{g}+\alpha / 2$ implies that $\underline{w} \geq|\underline{u}|$. Using the fact that

$$
\|u\|^{2}:=\int_{M}\left\{\left(\Delta_{g} u+\frac{\alpha}{2} u\right)^{2}+\left(\beta-\frac{\alpha^{2}}{4}\right) u^{2}\right\} d v_{g}, \quad \forall u \in H_{2}^{2}(M),
$$

and that $\beta-\alpha^{2} / 4 \leq 0$, we get

$$
\|\underline{w}\| \leq\|\underline{u}\| .
$$

Using Lemma 2.2, we conclude that

$$
E_{\lambda}(\underline{t}(\underline{w}, \lambda) \underline{w}) \leq E_{\lambda}(\underline{t}(\underline{u}, \lambda) \underline{u}) .
$$

We obtain that $\underline{t}(\underline{w}, \lambda) \underline{w}$ is a nontrivial nonnegative solution to (1.14). Finally, since $\underline{w} \geq 0, \underline{w} \neq 0$, then, applying again the maximum principle for the operator $\Delta_{g}+\alpha / 2$, we deduce that $\underline{w}$ is positive. The same arguments can be used for $\bar{v}_{n}$ to obtain a second positive solution $\bar{t}(\bar{w}, \lambda) \bar{w}$, which achieves the proof.

2.3. Behavior of the energy. In this subsection, we give results concerning the sign of the energy for the positive solutions to (1.14). To be more precise in the sequel, $\underline{t}\left(\underline{w}_{\mu}, \mu\right) \underline{w}_{\mu}$ and $\bar{t}\left(\bar{w}_{\mu}, \mu\right) \bar{w}_{\mu}$ will stand for the positive solutions found above, when the value of the parameter $\lambda$ is equal to $\mu$.

THEOREM 2.6. Let $1<q<2<r<2^{\#}$. Then

(a) $E_{\lambda}\left(\underline{t}\left(\underline{w}_{\lambda}, \lambda\right) \underline{w}_{\lambda}\right)<0$ for $\left.\lambda \in\right] 0, \hat{\lambda}[$,

(b) $E_{\lambda}\left(\bar{t}\left(\bar{w}_{\lambda}, \lambda\right) \bar{w}_{\lambda}\right)>0$ for $\left.\lambda \in\right] 0, \lambda_{0}\left[, E_{\lambda}\left(\bar{t}\left(\bar{w}_{\lambda}, \lambda\right) \bar{w}_{\lambda}\right)<0\right.$ for $\left.\lambda \in\right] \lambda_{0}, \hat{\lambda}[$, where

$$
\lambda_{0}:=\frac{q}{r}\left(\frac{r}{2}\right)^{(r-q) /(r-2)} \hat{\lambda}
$$

Proof. (a) We recall that $\partial_{t} \widetilde{E}_{\lambda}\left(\underline{t}\left(\underline{w}_{\lambda}, \lambda\right), \underline{w}_{\lambda}\right)=0$ and $\partial_{t t} \widetilde{E}_{\lambda}\left(\underline{t}\left(\underline{w}_{\lambda}, \lambda\right), \underline{w}_{\lambda}\right)>0$. Then

$$
\begin{gathered}
P\left(\underline{t}\left(\underline{w}_{\lambda}, \lambda\right) \underline{w}_{\lambda}\right)-\lambda Q\left(\underline{t}\left(\underline{w}_{\lambda}, \lambda\right) \underline{w}_{\lambda}\right)-R\left(\underline{t}\left(\underline{w}_{\lambda}, \lambda\right) \underline{w}_{\lambda}\right)=0, \\
P\left(\underline{t}\left(\underline{w}_{\lambda}, \lambda\right) \underline{w}_{\lambda}\right)-\lambda(q-1) Q\left(\underline{t}\left(\underline{w}_{\lambda}, \lambda\right) \underline{w}_{\lambda}\right)-(r-1) R\left(\underline{t}\left(\underline{w}_{\lambda}, \lambda\right) \underline{w}_{\lambda}\right)>0 .
\end{gathered}
$$

Using the fact that $1<q<2<r$, we get

$$
\left.\left.\left.\frac{1}{2} P\left(\underline{t}^{(} \underline{w}_{\lambda}, \lambda\right) \underline{w}_{\lambda}\right)-\frac{\lambda}{q} Q\left(\underline{t}^{(} \underline{w}_{\lambda}, \lambda\right) \underline{w}_{\lambda}\right)-\frac{1}{r} R\left(\underline{t}^{(} \underline{w}_{\lambda}, \lambda\right) \underline{w}_{\lambda}\right)<0,
$$

and consequently, $E_{\lambda}\left(\underline{t}\left(\underline{w}_{\lambda}, \lambda\right) \underline{w}_{\lambda}\right)<0$.

(b) Let $u$ be an arbitrary element of $H_{2}^{2}(M) \backslash\{0\}$ and we write

$$
\tilde{E}_{\lambda}(t, u)=t^{q} \widetilde{G}_{\lambda}(t, u) \text {, where } \widetilde{G}_{\lambda}(t, u)=t^{2-q} \frac{P(u)}{2}-\lambda \frac{Q(u)}{q}-t^{r-q} \frac{R(u)}{r} .
$$


It follows that

$$
\partial_{t} \widetilde{E}_{\lambda}(t, u)=q t^{q-1} \widetilde{G}_{\lambda}(t, u)+t^{q} \partial_{t} \widetilde{G}_{\lambda}(t, u),
$$

with

$$
\partial_{t} \widetilde{G}_{\lambda}(t, u)=t^{2-q-1}\left\{\frac{2-q}{2} P(u)-\frac{r-q}{r} t^{r-2} R(u)\right\}
$$

The real-valued function $t \mapsto \widetilde{G}_{\lambda}(t, u)$ is increasing on $] 0, t_{0}(u)$ [, decreasing on ] $t_{0}(u)$, $+\infty$ [, and reaches its unique maximum for $t=t_{0}(u)$, where

$$
t_{0}(u)=\left(\frac{r}{2}\right)^{1 /(r-2)} t(u)
$$

and $t(u)$ is defined in (2.5). On the other hand, a direct computation gives

$$
\tilde{G}_{\lambda}\left(t_{0}(u), u\right)=\left(\frac{2-q}{r-q} \frac{P(u)}{R(u)}\right)^{(r-q) /(r-2)} R(u)-\lambda Q(u) .
$$

Similarly, $\tilde{G}_{\lambda}\left(t_{0}(u), u\right)>0$ (resp., $\tilde{G}_{\lambda}\left(t_{0}(u), u\right)<0$ ) if $\lambda<\lambda_{0}(u)\left(\right.$ resp., $\left.\lambda>\lambda_{0}(u)\right)$ and $\widetilde{G}_{\lambda_{0}(u)}\left(t_{0}(u), u\right)=0$, where

$$
\lambda_{0}(u)=\frac{q}{r}\left(\frac{r}{2}\right)^{(r-q) /(r-2)} \lambda(u)
$$

and $\lambda(u)$ is given by (2.8). Thus, we get

$$
\begin{array}{ll}
\widetilde{E}_{\lambda}\left(t_{0}(u), u\right)>0 & \text { if } \lambda<\lambda_{0}(u), \\
\widetilde{E}_{\lambda}\left(t_{0}(u), u\right)=0 & \text { if } \lambda=\lambda_{0}(u), \\
\tilde{E}_{\lambda}\left(t_{0}(u), u\right)<0 & \text { if } \lambda>\lambda_{0}(u) .
\end{array}
$$

We consider the increasing real-valued function

$$
] 0,1\left[\longrightarrow \mathbb{R}, \quad t \longmapsto \frac{\ln (t)}{1-t}\right.
$$

Then, for every two real numbers $x, y$ such that $0<x<y<1$, one has

$$
\ln \left(\frac{1}{x}\right)>\frac{1-x}{1-y} \ln \left(\frac{1}{y}\right)=\ln \left(\left(\frac{1}{y}\right)^{(1-x) /(1-y)}\right)
$$

Therefore,

$$
0<x\left(\frac{1}{y}\right)^{(1-x) /(1-y)}<1
$$


In the specific case, $x=q / r$ and $y=2 / r$, we get

$$
0<\frac{q}{r}\left(\frac{r}{2}\right)^{(r-q) /(r-2)}<1
$$

which gives $0<\lambda_{0}(u)<\lambda(u)$.

Moreover, for every $u \in H_{2}^{2}(M) \backslash\{0\}$, one has $\widetilde{G}_{\lambda_{0}(u)}(t, u)<0$ for $\left.t \in\right] 0,+\infty\left[\backslash\left\{t_{0}(u)\right\}\right.$ and $\widetilde{G}_{\lambda_{0}(u)}\left(t_{0}(u), u\right)=0$. Hence, the real-valued function $t \mapsto \widetilde{E}_{\lambda_{0}(u)}(t, u), t>0$, reaches its unique maximum at $t=t_{0}(u)$ and we obtain the following relation:

$$
\bar{t}\left(u, \lambda_{0}(u)\right)=t_{0}(u) .
$$

Classical arguments of variational calculus show that $\lambda_{0}(u)$ is weakly lower semicontinuous on $H_{2}^{2}(M) \backslash\{0\}$. Then, the value

$$
\lambda_{0}:=\inf _{u \in H_{2}^{2}(M) \backslash\{0\}} \lambda_{0}(u)
$$

is achieved on $H_{2}^{2}(M) \backslash\{0\}$. Since $\lambda_{0}(u)$ is homogeneous in $u$, we can assume that there is some $u^{*} \in \mathbb{S}$ such that $\lambda_{0}=\lambda_{0}\left(u^{*}\right)$.

Now, let $\lambda \in] 0, \lambda_{0}$ [. Then, for every $u \in H_{2}^{2}(M) \backslash\{0\}$, one has $\lambda<\lambda_{0}(u)$, and consequently $\widetilde{E}_{\lambda}\left(t_{0}(u), u\right)>0$ holds true from (2.75). But, $t \mapsto \widetilde{E}_{\lambda}(t, u), t>0$, reaches its unique maximum for $t=\bar{t}(u, \lambda)$, hence $\widetilde{E}_{\lambda}(\bar{t}(u, \lambda), u)>0$, for every $u \in H_{2}^{2}(M) \backslash\{0\}$. In particular, we have $\widetilde{E}_{\lambda}\left(\bar{t}\left(\bar{w}_{\lambda}, \lambda\right), \bar{w}_{\lambda}\right)>0$, that is, $E_{\lambda}\left(\bar{t}\left(\bar{w}_{\lambda}, \lambda\right) \bar{w}_{\lambda}\right)>0$. In the specific case $\lambda=\lambda_{0}$, one has

$$
\begin{aligned}
E_{\lambda_{0}}\left(\bar{t}\left(\bar{w}_{\lambda_{0}}, \lambda_{0}\right) \bar{w}_{\lambda_{0}}\right) & =\widetilde{E}_{\lambda_{0}}\left(\bar{t}\left(\bar{w}_{\lambda_{0}}, \lambda_{0}\right), \bar{w}_{\lambda_{0}}\right) \\
& =\inf _{u \in \mathbb{S}} \widetilde{E}_{\lambda_{0}}\left(\bar{t}\left(u, \lambda_{0}\right), u\right) \\
& \leq \widetilde{E}_{\lambda_{0}}\left(\bar{t}\left(u^{*}, \lambda_{0}\left(u^{*}\right)\right), u^{*}\right) \\
& =\widetilde{E}_{\lambda_{0}\left(u^{*}\right)}\left(t_{0}\left(u^{*}\right), u^{*}\right) \\
& =0 .
\end{aligned}
$$

This implies that $E_{\lambda_{0}}\left(\bar{t}\left(\bar{w}_{\lambda_{0}}, \lambda_{0}\right) \bar{w}_{\lambda_{0}}\right) \leq 0$. Thanks to (2.75), we have, in addition, $\widetilde{E}_{\lambda_{0}}\left(t_{0}(u), u\right) \geq 0$ and $\widetilde{E}_{\lambda_{0}}\left(\underline{t}\left(u, \lambda_{0}\right), u\right)<0$, for every $u \in H_{2}^{2}(M) \backslash\{0\}$. Then, it comes that

$$
\begin{gathered}
t_{0}(u)>\underline{t}\left(u, \lambda_{0}\right), \quad \forall u \in H_{2}^{2}(M) \backslash\{0\}, \\
\widetilde{E}_{\lambda_{0}}\left(\bar{t}\left(\bar{w}_{\lambda_{0}}, \lambda_{0}\right), \bar{w}_{\lambda_{0}}\right) \geq \widetilde{E}_{\lambda_{0}}\left(t_{0}\left(\bar{w}_{\lambda_{0}}\right), \bar{w}_{\lambda_{0}}\right) \geq 0 .
\end{gathered}
$$

Finally, one gets

$$
E_{\lambda_{0}}\left(\bar{t}\left(\bar{w}_{\lambda_{0}}, \lambda_{0}\right) \bar{w}_{\lambda_{0}}\right)=0
$$

Now, assume that $\lambda_{0}<\lambda<\hat{\lambda}$. Since for every $\left.(t, u) \in\right] 0,+\infty\left[\times\left(H_{2}^{2}(M) \backslash\{0\}\right)\right.$, the realvalued function $\lambda \mapsto \widetilde{E}_{\lambda}(t, u)$ is decreasing, it follows that

$$
\widetilde{E}_{\lambda}(t, u)<\widetilde{E}_{\lambda_{0}}(t, u), \text { for every } t>0, u \in H_{2}^{2}(M) \backslash\{0\} .
$$


In addition, we have

$$
\begin{aligned}
\widetilde{E}_{\lambda}\left(\bar{t}\left(\bar{w}_{\lambda}, \lambda\right), \bar{w}_{\lambda}\right) & =\inf _{u \in \mathbb{S}} \widetilde{E}_{\lambda}(\bar{t}(u, \lambda), u) \\
& \leq \widetilde{E}_{\lambda}\left(\bar{t}\left(u^{*}, \lambda\right), u^{*}\right) \\
& <\widetilde{E}_{\lambda_{0}}\left(\bar{t}\left(u^{*}, \lambda\right), u^{*}\right),
\end{aligned}
$$

where the last inequality follows from (2.85). Moreover, the real-valued function $t \mapsto$ $\widetilde{E}_{\lambda_{0}}\left(t, u^{*}\right), t>0$, achieves its global maximum at $t=t_{0}\left(u^{*}\right)$. Thus, $\widetilde{E}_{\lambda_{0}}\left(\bar{t}\left(u^{*}, \lambda\right), u^{*}\right) \leq$ $\widetilde{E}_{\lambda_{0}}\left(t_{0}\left(u^{*}\right), u^{*}\right)=\widetilde{E}_{\lambda_{0}\left(u^{*}\right)}\left(t_{0}\left(u^{*}\right), u^{*}\right)=0$. Hence, $E_{\lambda}\left(\bar{t}\left(\bar{w}_{\lambda}, \lambda\right) \bar{w}_{\lambda}\right)<0$, which ends the proof.

The following result shows that the variational character of (2.13) has a genuine link with the main problem (1.14).

THEOREM 2.7. If $u$ is a solution of (2.81), then $t_{0}(u) u$ is a solution of (1.14) for $\lambda=\lambda_{0}$.

Proof. If $u$ is a solution of (2.81), then $\lambda_{0}=\lambda_{0}(u)$ and for every $\varphi \in H_{2}^{2}(M)$, we have

$$
\begin{aligned}
E_{\lambda_{0}}^{\prime}\left(t_{0}(u) u\right)(\varphi) & =\frac{1}{2} P^{\prime}\left(t_{0}(u) u\right)(\varphi)-\frac{\lambda_{0}}{q} Q^{\prime}\left(t_{0}(u) u\right)(\varphi)-\frac{1}{r} R^{\prime}\left(t_{0}(u) u\right)(\varphi) \\
& =\frac{P(u)\left[t_{0}(u)\right]}{2}\left(\frac{P^{\prime}(u)(\varphi)}{P(u)}-\frac{r-2}{r-q} \frac{Q^{\prime}(u)(\varphi)}{Q(u)}-\frac{2-q}{r-q} \frac{R^{\prime}(u)(\varphi)}{R(u)}\right) \\
& =K\left(\frac{r-q}{r-2} \frac{P^{\prime}(u)(\varphi)}{P(u)}-\frac{Q^{\prime}(u)(\varphi)}{Q(u)}-\frac{2-q}{r-2} \frac{R^{\prime}(u)(\varphi)}{R(u)}\right),
\end{aligned}
$$

where

$$
K:=\frac{r-2}{r-q} \frac{P(u)}{2}\left[t_{0}(u)\right]
$$

By hypothesis, one gets $\lambda_{0}^{\prime}(u)(\varphi)=0$ for every $\varphi \in H_{2}^{2}(M)$, and

$$
\lambda_{0}^{\prime}(u)(\varphi)=\lambda_{0}\left(\frac{r-q}{r-2} \frac{P^{\prime}(u)(\varphi)}{P(u)}-\frac{Q^{\prime}(u)(\varphi)}{Q(u)}-\frac{2-q}{r-2} \frac{R^{\prime}(u)(\varphi)}{R(u)}\right) .
$$

We conclude that

$$
E_{\lambda_{0}}^{\prime}\left(t_{0}(u) u\right)(\varphi)=\frac{K}{\lambda_{0}} \lambda_{0}^{\prime}(u)(\varphi)=0
$$

for every $\varphi \in H_{2}^{2}(M)$, which implies that $t_{0}(u) u$ is a solution to (1.14) for $\lambda=\lambda_{0}$.

3. Infinitely many solutions. In this section, we show the existence of infinitely many solutions to (1.14). More precisely, we carry out two disjoint and infinite sets of solutions to (1.14). One set consists of solutions with negative energy, while the other set contains solutions with arbitrary energy. We briefly recall here some background facts that we will use in the sequel [20, 21, 24, 26]. Let

$$
\mathscr{A}=\{A \subset \mathbb{S}: A \text { closed, } A=-A\}
$$


be the class of closed and symmetric subsets of the complete smooth submanifold $(\mathbb{S},\|\cdot\|)$. For every $A \in \mathscr{A}, A \neq \varnothing$, let

$$
\gamma(A)=\inf \left\{k \in \mathbb{N} ; \exists \varphi \in C^{0}\left(A, \mathbb{R}^{k} \backslash\{0\}\right), \forall u \in A, \varphi(-u)=-\varphi(u)\right\}
$$

be the Krasnoselskii genus [20]. When there does not exist a finite such integer, set $\gamma(A)=+\infty$. Finally, set $\gamma(\varnothing)=0$. For each positive integer $k$, we define

$$
\begin{gathered}
\Gamma_{k}=\{A \in \mathscr{A} ; A \text { compact, } \gamma(A) \geq k\}, \\
\underline{c}_{k}=\inf _{A \in \Gamma_{k}} \max _{u \in A} \underline{\mathscr{E}}_{\lambda}(u), \quad \overline{\mathcal{c}}_{k}=\inf _{A \in \Gamma_{k}} \max _{u \in A} \overline{\mathscr{E}}_{\lambda}(u),
\end{gathered}
$$

where

$$
\begin{aligned}
& \underline{\mathscr{E}}_{\lambda}(u)=E_{\lambda}(\underline{t}(u, \lambda) u), \\
& \overline{\mathscr{E}}_{\lambda}(u)=E_{\lambda}(\bar{t}(u, \lambda) u) .
\end{aligned}
$$

It is well known that $\left(\underline{c}_{k}\right)$ (resp., $\left.\left(\bar{c}_{k}\right)\right)$ is a nondecreasing sequence of critical values of $\underline{\mathscr{E}}_{\lambda}$ (resp., $\left.\overline{\mathscr{E}}_{\lambda}\right)$ [26]. Recall that if the sequence $\left(\underline{c}_{k}\right)$ (resp., $\left.\left(\bar{c}_{k}\right)\right)$ is increasing, then $\underline{\mathscr{E}}_{\lambda}$ (resp., $\left.\overline{\mathscr{E}}_{\lambda}\right)$ has infinitely many critical points $\left(\underline{u}_{\lambda, k}\right)$ (resp., $\left(\bar{u}_{\lambda, k}\right)$ ) corresponding to the sequence of distinct levels $\left(\underline{c}_{k}\right)$ (resp., $\left(\bar{c}_{k}\right)$ ). On the other hand, if there are two positive integers $j$ and $p$ such that $\underline{c}_{j}=\underline{c}_{j+1}=\cdots=\underline{c}_{j+p}$ (resp., $\bar{c}_{j}=\bar{c}_{j+1}=\cdots=\bar{c}_{j+p}$ ), then the set of critical points for $\underline{\mathscr{E}}_{\lambda}$ (resp., $\overline{\mathscr{E}}_{\lambda}$ ) corresponding to the level $\underline{c}_{j}$ (resp., $\bar{c}_{j}$ ) is infinite. Hereafter, we set

$$
\begin{aligned}
& \underline{v}_{\lambda, k}=\underline{t}\left(\underline{u}_{\lambda, k}, \lambda\right) \underline{u}_{\lambda, k}, \\
& \bar{v}_{\lambda, k}=\bar{t}\left(\bar{u}_{\lambda, k}, \lambda\right) \bar{u}_{\lambda, k} .
\end{aligned}
$$

We recall that $\underline{v}_{\lambda, k}$ and $\bar{v}_{\lambda, k}$ are solutions to (1.14) for every $k \in \mathbb{N}^{*}$.

THEOREM 3.1. Let $1<q<2<r<2^{\#}$ and $0<\lambda<\hat{\lambda}$. Then there are two disjoint and infinite sets of solutions to (1.14): $\left\{\underline{v}_{\lambda, k} ; k \in \mathbb{N}^{*}\right\}$ and $\left\{\bar{v}_{\lambda, k} ; k \in \mathbb{N}^{*}\right\}$. In addition,

(a) $\lim _{k \rightarrow+\infty} E_{\lambda}\left(\bar{v}_{\lambda, k}\right)=+\infty$,

(b) $E_{\lambda}\left(\underline{v}_{\lambda, k}\right)<0, \lim _{k \rightarrow+\infty} E_{\lambda}\left(\underline{v}_{\lambda, k}\right)=0$.

Proof. We recall that $\partial_{t t} \widetilde{E}_{\lambda}\left(\underline{t}\left(\underline{u}_{\lambda, k}, \lambda\right), \underline{u}_{\lambda, k}\right)>0$ and $\partial_{t t} \widetilde{E}_{\lambda}\left(\bar{t}\left(\bar{u}_{\lambda, k}, \lambda\right), \bar{u}_{\lambda, k}\right)<0$. Then the two sets $\left\{\underline{v}_{\lambda, k} ; k \in \mathbb{N}^{*}\right\}$ and $\left\{\bar{v}_{\lambda, k} ; k \in \mathbb{N}^{*}\right\}$ are disjoint.

(a) As mentioned above, the sequence $\left(\bar{c}_{k}\right):=\left(\overline{\mathscr{E}}_{\lambda}\left(\bar{u}_{\lambda, k}\right)\right):=\left(E_{\lambda}\left(\bar{v}_{\lambda, k}\right)\right)$ is nondecreasing. Suppose, by contradiction, that

$$
\lim _{k \rightarrow \infty} \bar{c}_{k}=\bar{c}<+\infty
$$

and consider the symmetric set

$$
K_{\bar{c}}=\left\{u \in \mathbb{S} ; \overline{\mathscr{E}}_{\lambda}(u)=\bar{c}, \overline{\mathscr{C}}_{\lambda}^{\prime}(u)=0\right\} .
$$


It is easy to see that

$$
\begin{gathered}
\lim _{k \rightarrow \infty} E_{\lambda}\left(\bar{t}\left(\bar{u}_{\lambda, k}, \lambda\right) \bar{u}_{\lambda, k}\right)=\bar{c}, \\
E_{\lambda}^{\prime}\left(\bar{t}\left(\bar{u}_{\lambda, k}, \lambda\right) \bar{u}_{\lambda, k}\right)=0 .
\end{gathered}
$$

Since $E_{\lambda}$ satisfies the Palais-Smale condition, we conclude that $K_{\bar{c}}$ is not empty and compact. Then $\gamma\left(K_{\bar{c}}\right)<+\infty$ [24]. Let $N$ be a closed neighborhood of $K_{\bar{c}}$ in $\mathbb{S}$ such that $\gamma(N)=\gamma\left(K_{\bar{c}}\right)$. The deformation lemma [21, 24, 26, 28] ensures the existence of an odd homeomorphism $\Phi$ from $\mathbb{S}$ to $\mathbb{S}$ and $\varepsilon>0$ such that

$$
\Phi\left(A_{\bar{C}+\varepsilon} \backslash \stackrel{\circ}{N}\right) \subset A_{\bar{C}-\varepsilon}
$$

Using classical properties of the Krasnoselskii genus, one gets

$$
\begin{aligned}
\gamma\left(A_{\overline{\mathcal{C}}+\varepsilon}\right) & \leq \gamma\left(A_{\overline{\bar{c}}+\varepsilon} \backslash \stackrel{\circ}{N}\right)+\gamma(N) \\
& \leq \gamma\left(\Phi\left(A_{\overline{\mathcal{C}}+\varepsilon} \backslash \stackrel{\circ}{N}\right)\right)+\gamma(N) \\
& \leq \gamma\left(A_{\overline{\mathcal{C}}-\varepsilon}\right)+\gamma(N) .
\end{aligned}
$$

On the other hand, by the definition of $\bar{c}$, there is a positive integer $j$ such that $\bar{c}-\varepsilon<$ $c_{j} \leq \bar{c}$. As a consequence, one gets $\gamma\left(A_{\bar{c}-\varepsilon}\right)<j$ and

$$
\gamma\left(A_{\bar{c}+\varepsilon}\right)<j+\gamma\left(K_{\bar{c}}\right)<+\infty .
$$

But this is in contradiction with $\gamma\left(A_{\bar{c}+\varepsilon}\right)=+\infty$. We finally obtain that

$$
\lim _{k \rightarrow \infty} \bar{c}_{k}=+\infty
$$

(b) It is known from the above that $E_{\lambda}\left(\underline{v}_{\lambda, k}\right)<0$ for every $k$. We show that $\left(\underline{c}_{k}\right)$ converges to zero as $k$ goes to infinity. Suppose that

$$
\lim _{k \rightarrow+\infty} \underline{c}_{k}=\underline{c}<0
$$

As before, consider the symmetric set

$$
K_{\underline{c}}=\left\{u \in \mathbb{S} ; \underline{\mathscr{E}}_{\lambda}(u)=\underline{c}, \underline{\mathscr{E}}_{\lambda}^{\prime}(u)=0\right\} .
$$

Since $\underline{\mathscr{E}}$ satisfies that Palais-Smale condition on $\mathbb{S}$ and $\underline{c}<0$, then $K_{\underline{c}}$ is not empty and compact, which implies that $\gamma\left(K_{\underline{\mathcal{C}}}\right)<+\infty$. Let $N$ be a closed neighborhood of $K_{\underline{\underline{c}}}$ in $\mathbb{S}$ such that $\gamma(N)=\gamma\left(K_{\underline{c}}\right)$. Applying again the deformation lemma, there are an odd homeomorphism $\Psi$ from $\mathbb{S}$ to $\mathbb{S}$ and $\varepsilon>0$ such that

$$
\Psi\left(A_{\underline{\underline{c}}+\varepsilon} \backslash \stackrel{\circ}{N}\right) \subset A_{\underline{\underline{c}}-\varepsilon}
$$


As mentioned above, we get

$$
\begin{aligned}
\gamma\left(A_{\underline{\mathcal{C}}+\varepsilon}\right) & \leq \gamma\left(A_{\underline{\underline{c}}+\varepsilon} \backslash \stackrel{\circ}{N}\right)+\gamma(N) \\
& \leq \gamma\left(\Psi\left(A_{\underline{\mathcal{c}}+\varepsilon} \backslash \stackrel{\circ}{N}\right)\right)+\gamma(N) \\
& \leq \gamma\left(A_{\underline{\underline{c}}-\varepsilon}\right)+\gamma(N) .
\end{aligned}
$$

Furthermore, there is a positive integer $j$ such that $\underline{c}-\varepsilon<c_{j} \leq \underline{c}$; then $\gamma\left(A_{\underline{c}-\varepsilon}\right)<j$, and consequently

$$
\gamma\left(A_{\underline{c}+\varepsilon}\right)<j+\gamma\left(K_{\underline{c}}\right)<+\infty .
$$

This contradicts $\gamma\left(A_{\underline{\underline{C}}+\varepsilon}\right)=+\infty$, which ends the proof.

Comment. Consider the specific case where the functions $a$ and $b$ are positive constants. Then problem (1.14) possesses two constant and positive solutions $\underline{c}(\lambda)$ and $\bar{c}(\lambda)$. The solutions $\underline{c}(\lambda)$ and $\bar{c}(\lambda)$ realize, respectively, the (unique) local minimum and the (unique) local maximum of the real-valued function

$$
\frac{\beta}{2} t^{2}-\lambda \frac{a}{q} t^{q}-\frac{b}{r} t^{r}, \quad t>0,
$$

where

$$
0<\lambda<\lambda^{*}:=\hat{C} \frac{\beta^{(r-q) /(r-2)}}{a b^{(2-q) /(r-2)}} .
$$

Notice that $\hat{\lambda} \leq \lambda^{*}$. An interesting question is to compare $\underline{\alpha}(\lambda)$ (resp., $\bar{\alpha}(\lambda)$ ) with $E_{\lambda}(\underline{c}(\lambda))$ (resp., with $E_{\lambda}(\bar{c}(\lambda))$ ). If, for example, $\underline{\alpha}(\lambda)<E_{\lambda}(\underline{c}(\lambda))$ and $\bar{\alpha}(\lambda)<E_{\lambda}(\bar{c}(\lambda))$, then (1.14) possesses four positive solutions.

ACKNOWLEDgment. The author is very grateful to Professor Emmanuel Hebey for stimulating discussions and helpful comments on the results.

\section{REFERENCES}

[1] A. Ambrosetti, H. Brézis, and G. Cerami, Combined effects of concave and convex nonlinearities in some elliptic problems, J. Funct. Anal. 122 (1994), no. 2, 519-543.

[2] A. Ambrosetti, J. Garcia Azorero, and I. Peral, Existence and multiplicity results for some nonlinear elliptic equations: a survey, Rend. Mat. Appl. (7) 20 (2000), 167-198.

[3] W. Beckner, Sharp Sobolev inequalities on the sphere and the Moser-Trudinger inequality, Ann. of Math. (2) 138 (1993), no. 1, 213-242.

[4] F. Bernis, J. Garcia Azorero, and I. Peral, Existence and multiplicity of nontrivial solutions in semilinear critical problems of fourth order, Adv. Differential Equations 1 (1996), no. 2, 219-240.

[5] T. P. Branson, Group representations arising from Lorentz conformal geometry, J. Funct. Anal. 74 (1987), no. 2, 199-291.

[6] T. P. Branson, S.-Y. A. Chang, and P. C. Yang, Estimates and extremals for zeta function determinants on four-manifolds, Comm. Math. Phys. 149 (1992), no. 2, 241-262.

[7] H. Brézis and E. Lieb, A relation between pointwise convergence of functions and convergence of functionals, Proc. Amer. Math. Soc. 88 (1983), no. 3, 486-490. 
[8] S.-Y. A. Chang, M. J. Gursky, and P. C. Yang, Regularity of a fourth order nonlinear PDE with critical exponent, Amer. J. Math. 121 (1999), no. 2, 215-257.

[9] S.-Y. A. Chang and P. C. Yang, Extremal metrics of zeta function determinants on 4manifolds, Ann. of Math. (2) 142 (1995), no. 1, 171-212.

[10] _ On a fourth order curvature invariant, Spectral Problems in Geometry and Arithmetic (Iowa, 1997) (T. Branson, ed.), Contemp. Math., vol. 237, American Mathematical Society, Rhode Island, 1999, pp. 9-28.

[11] Z. Djadli, E. Hebey, and M. Ledoux, Paneitz-type operators and applications, Duke Math. J. 104 (2000), no. 1, 129-169.

[12] P. Drábek and S. I. Pohozaev, Positive solutions for the $p$-Laplacian: application of the fibering method, Proc. Roy. Soc. Edinburgh Sect. A 127 (1997), no. 4, 703-726.

[13] A. El Hamidi, Multiple solutions with changing sign energy to a nonlinear elliptic equation, Commun. Pure Appl. Anal. 3 (2004), no. 2, 253-265.

[14] P. Esposito and F. Robert, Mountain pass critical points for Paneitz-Branson operators, Calc. Var. Partial Differential Equations 15 (2002), no. 4, 493-517.

[15] V. Felli, E. Hebey, and F. Robert, Fourth order equations of critical Sobolev growth. Energy function and solutions of bounded energy in the conformally flat case, to appear in NoDEA Nonlinear Differential Equations Appl.

[16] M. J. Gursky, The Weyl functional, de Rham cohomology, and Kähler-Einstein metrics, Ann. of Math. (2) 148 (1998), no. 1, 315-337.

[17] E. Hebey, Fourth order partial differential equations on compact Riemannian manifolds, preprint, 2003.

[18] _ _ Sharp Sobolev inequalities of second order, J. Geom. Anal. 13 (2003), no. 1, 145162.

[19] E. Hebey and F. Robert, Coercivity and Struwe's compactness for Paneitz type operators with constant coefficients, Calc. Var. Partial Differential Equations 13 (2001), no. 4, 491-517.

[20] M. A. Krasnosel'skii, Topological Methods in the Theory of Nonlinear Integral Equations, The Macmillan, New York, 1964.

[21] J. Mawhin and M. Willem, Critical Point Theory and Hamiltonian Systems, Applied Mathematical Sciences, vol. 74, Springer-Verlag, New York, 1989.

[22] Z. Nehari, On a class of nonlinear second-order differential equations, Trans. Amer. Math. Soc. 95 (1960), 101-123.

[23] S. Paneitz, A quartic conformally covariant differential operator for arbitrary pseudoRiemannian manifolds, preprint, 1983.

[24] P. H. Rabinowitz, Minimax Methods in Critical Point Theory with Applications to Differential Equations, CBMS Regional Conference Series in Mathematics, vol. 65, American Mathematical Society, Rhode Island, 1986.

[25] F. Robert, Positive solutions for a fourth order equation invariant under isometries, Proc. Amer. Math. Soc. 131 (2003), no. 5, 1423-1431.

[26] M. Struwe, Variational Methods. Applications to Nonlinear Partial Differential Equations and Hamiltonian Systems, Ergebnisse der Mathematik und ihrer Grenzgebiete (3), vol. 34, Springer-Verlag, Berlin, 1996.

[27] R. C. A. M. Van der Vorst, Fourth-order elliptic equations with critical growth, C. R. Acad. Sci. Paris Sér. I Math. 320 (1995), no. 3, 295-299.

[28] M. Willem, Minimax Theorems, Progress in Nonlinear Differential Equations and Their Applications, 24, Birkhäuser Boston, Massachusetts, 1996.

Abdallah El Hamidi: Laboratoire de Mathématiques et Applications, Université de La Rochelle, avenue Michel Crépeau, 17000 La Rochelle, France

E-mail address: ae1hamid@univ-1r.fr 


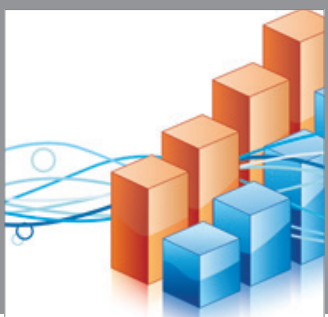

Advances in

Operations Research

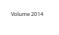

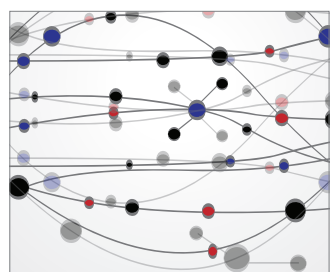

\section{The Scientific} World Journal
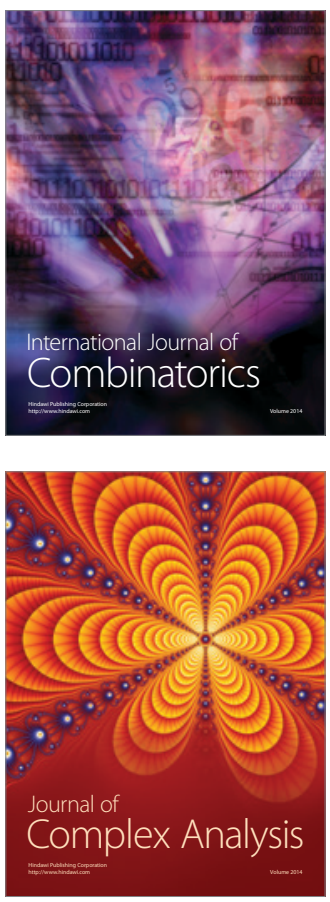

International Journal of

Mathematics and

Mathematical

Sciences
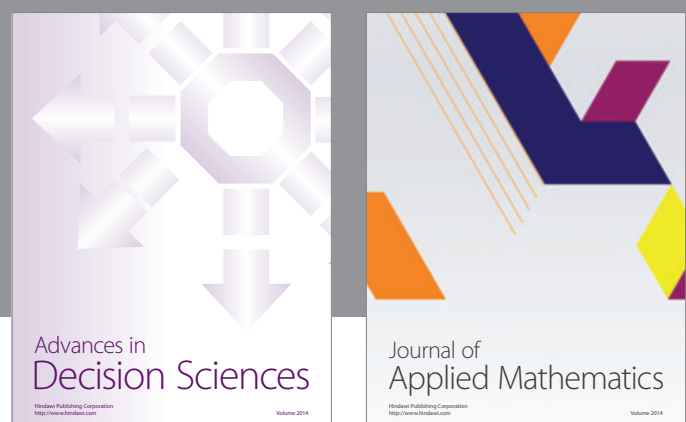

Journal of

Applied Mathematics
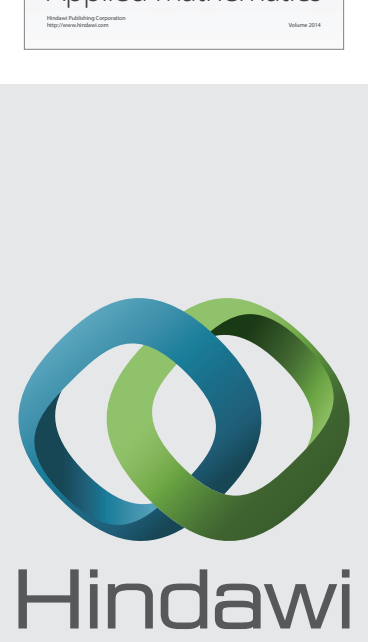

Submit your manuscripts at http://www.hindawi.com
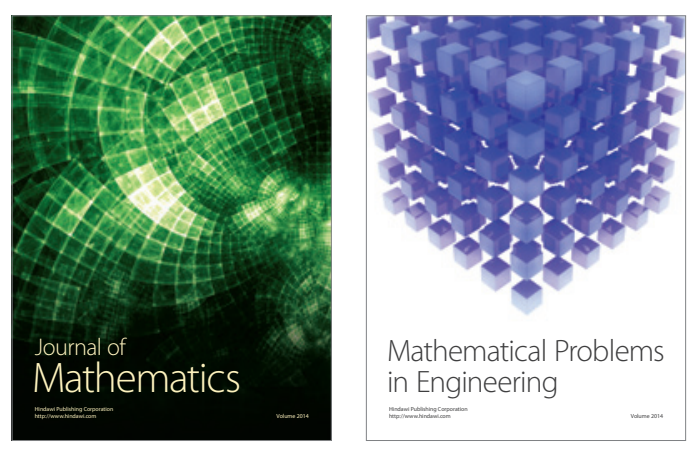

Mathematical Problems in Engineering
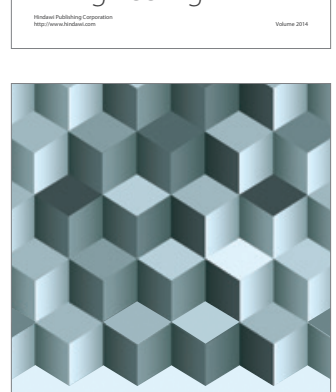

Journal of

Function Spaces
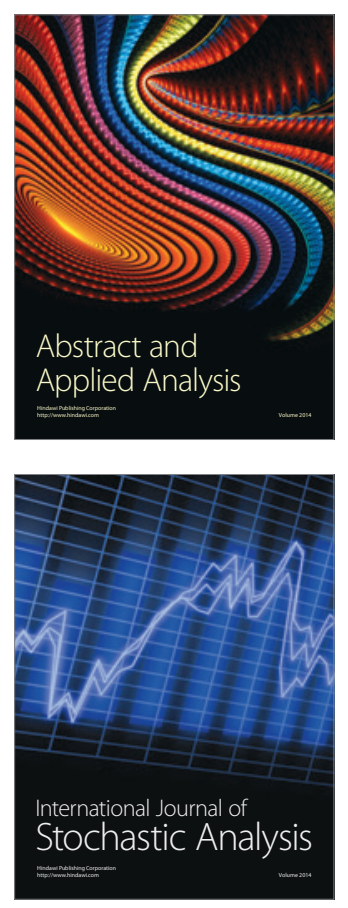

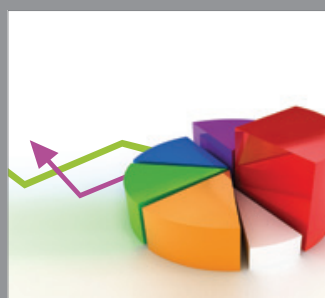

ournal of

Probability and Statistics

Promensencen
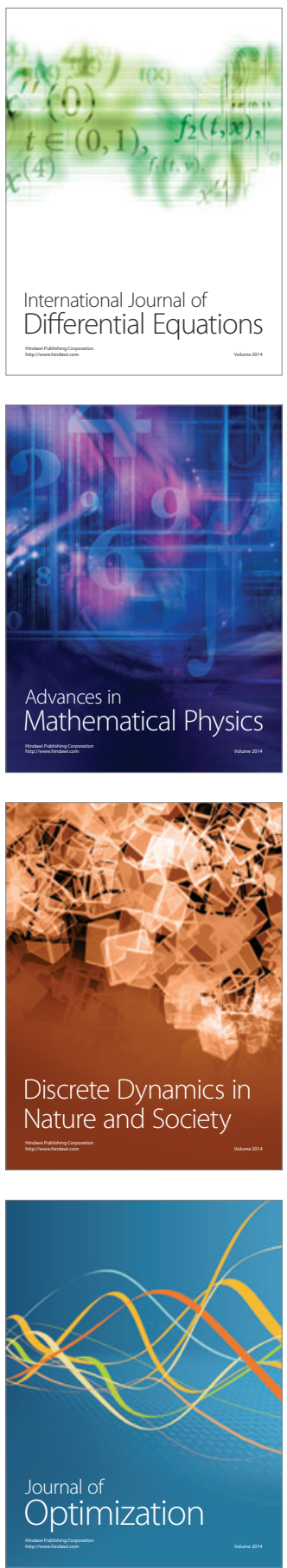